\title{
LA-UR- $96-3830$
}

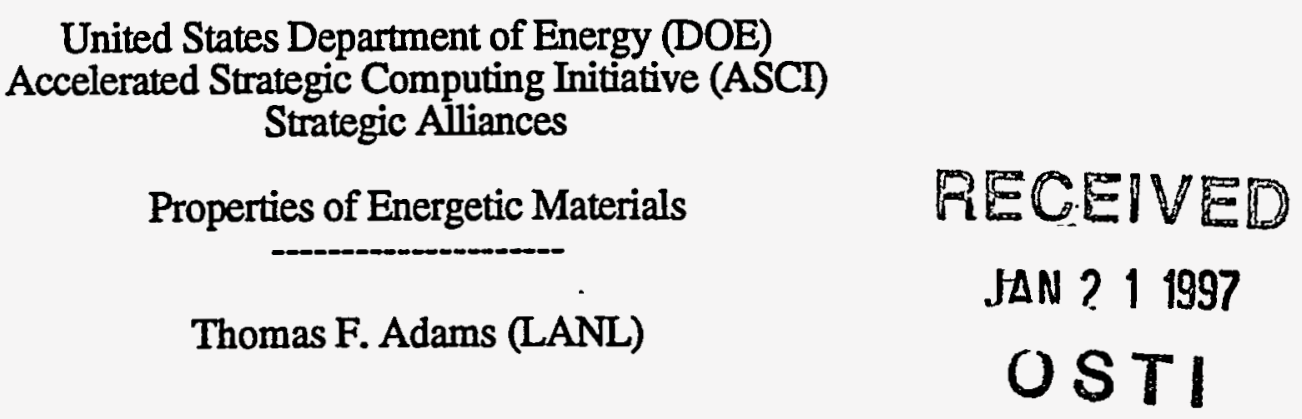

The Accelerated Strategic Computing Initiative (ASCI) program is designed to provide the computational resources which are required to provide a simulation based approach to the Science Based Stockpile Stewardship (SBSS) program. The capability to predict the properties of energetic materials is one of the areas of interest to the US Department of Energy's (DOE) ASCI program. This capability will support computational assessments of the safety and reliability of systems containing explosives and other energetic materials subjected to normal and abnormal environments.

Several research elements related to energetic material properties are described in more detail below. They are: (A) calculation of decomposition rates, (B) molecular potential functions, (C) physical properties and transport coefficients, (D) molecular energization mechanisms, (E) fracture/failure of energetic material crystals, (F) grain-grain and grainbinder interactions, and (G) aging effects in energetic materials. These elements have in common the need to develop computational methods that have a strong foundation in basic physical principles. They will generally have to be implemented to run efficiently on advanced parallel computing platforms to achieve sufficient accuracy.

\section{A. Calculation of decomposition rates}

Energetic materials, by their very nature, are metastable and wili decompose at varying rates under all conditions. For an ideal detonation, the chemical energy alone determines all the detonation conditions. Real explosives and energetic materials are not ideal. Their behavior is controlled by thermal chemistry. Reactive effects change all the properties of these materials, so we must understand the properties not just for the pristine state, but also as the reactions occur.

Quantum calculations of intramolecular potential energy surfaces are used to determine the reaction pathways. Molecular dynamics approaches are then used to describe how the condensed system modifies the rates associated with these pathways. Such calculations are widely done now for gas phase reactions. Determining the effect of the condensed state on the rates is the challenge.

\section{B. Molecular potential functions}

Molecular potential functions form the foundation for predicting the properties of energetic material systems. Simple (or fairly simple), transferable potential-energy functions parameterized for explosive and energetic molecules over a wide range of conditions are fundamental to modeling activities for weapons performance, safety, reliability, and renewal/manufacturing. Prediction of the performance of energetic materials during and after detonation necessitates interaction potentials accurate over a wide range of pressures. Modeling the response of energetic materials in a fire following a crash requires models applicable at extremely high temperatures. Predicting the impact of age on the performance

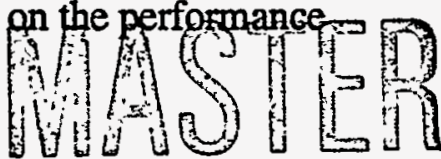




\section{DISCLAIMIER}

This report was prepared as an account of work sponsored by an agency of the United States Government. Neither the United States Government nor any agency thereof, nor any of their employees, makes any warranty, express or implied, or assumes any legal liability or responsibility for the accuracy, completeness, or usefulness of any information, apparatus, product, or process disclosed, or represents that its use would not infringe privately owned rights. Reference herein to any specific commercial product, process, or service by trade name, trademark, manufacturer, or otherwise does not necessarily constitute or imply its endorsement, recommendation, or favoring by the United States Government or any agency thereof. The views and opinions of authors expressed herein do not necessarily state or reflect those of the United States Government or any agency thereof. 


\section{DISCLAIMER}

Portions of this document may be illegible in electronic image products. Images are produced from the best available original document. 
and safety of energetic materials not only requires molecular interaction potentials accurate at high temperatures and widely varying pressures but also over the wide range of environments experience by a weapon during its lifetime (e.g. temperatures ranging from $120 \mathrm{~F}$ to $120 \mathrm{~F}$ ). Finally, modeling manufacturing processes as well as deriving new, improved energetic materials requires a fundamental understanding of the structureproperty relationships (nearly synonymous with accurate interaction potentials) of these materials under processing conditions as well as the performance and safety environments.

Potential-energy surfaces for "generic" crystals have been developed for hydrocarbons and azabenzenes (e.g., Williams et al., University of Kentucky). Potential energy surfaces for the most important molecules in high explosives and energetic materials, such as the nitramines and nitrated/aminated benzenes, may be calculable by extending the same methods to these molecules. In addition, the calibrations for these potential functions should be extended beyond ambient conditions to much higher temperatures and pressures to be relevant to explosive reaction zones.

\section{Calculations of physical properties and transport coefficients}

Physical properties and transport coefficients must be obtained to advance energetic material simulations from the molecular scale to larger distance and time scales. Starting from molecular potential functions it should be possible to provide, essentially from first principles, many of the required fundamental physical parameters. The physical properties that must be calculated include density, energy, specific heat, vectoral and scalar coefficients of thermal and isobaric expansion, elastic constants and derived moduli, mechanical strength, all as a function of temperature and pressure, for both solids and liquids. It is also important to develop practical methods to compute stress-strain curves and transport coefficients such as diffusion, viscosity, and thermal conductivity. These are harder to obtain due to the more complicated theoretical framework required.

A variety of theoretical tools are required to do these calculations. They include high-level $a b$ initio quantum electronic structure methods, Monte Carlo methods, and both classical and quantum mechanical molecular dynamics. Nearly the entire spectrum of modern computing capabilities must be brought to bear in this work, from high-performance workstations in stand-alone mode and in clusters, to advanced high-end parallel pla ${ }^{+}$orms.

\section{Simulations of molecular energization mechanisms}

Energization mechanisms must be simulated in order to determine the time required for thermal equilibration among the vibrational, rotational, and translational modes of the molecules in shocked/strained crystals of energetic material. This problem bears fairly strongly on questions about the degree to which microscopic (i.e., Angstrom- or nanometer-scale) initiation must be considered in modeling larger distance/time scale phenomena in energetic materials.

Some success has been achieved in a related area by Carter White et al. at NRL and Rice et al. at ARL who have simulated fully developed detonation waves in prototypical systems such as $2 \mathrm{AB} \rightarrow \mathrm{A}_{2}+\mathrm{B}_{2}$ and $2 \mathrm{O}_{3} \rightarrow 3 \mathrm{O}_{2}$. In contrast, the aim of this research element is not to simulate the detonation dynamics, but rather to ferret out the molecular-level details of the energization process in a shock front and the subsequent energy relaxation that occurs in the moments subsequent to shock passage but before widespread chemical reaction. 
Treating the dynamics of large groups of molecules requires the development of massively parallel methods to treat molecular systems rather than simple atomic materials. Although this follows the successful development of molecular dynamics codes for atomic systems, this significant extension will require the implementation of methods such as fast multipole algorithms capable of dealing with relatively long-range interactions between the atoms in different molecules. While the methods can be developed and tested initially on high-end workstations, the large-scale massively parallel molecular dynamics calculations will have to be performed on advanced high-end parallel platforms.

\section{E. Fracture/failure of energetic material crystals}

The need for this research element stems from the lack of understanding about the mechanism and/or strain-rate dependence of failure in explosive crystals under either compression or tension. The major difficulties that must be dealt with center around incorporation of molecular bonding, high-frequency vibrations, and the effects of longrange potentials on the required force and energy evaluations. These issues put limits on the temporal and spatial domains that can be treated. The ongoing work of Holian, et al., and Lomdahl, et al,, at Los Alamos in the area of atomistic fracture mechanics of metals provides a framework for the simulations that will have to be done. Initial modeling attempts will be inadequate to simulate hundreds of millions of atoms, but should be able to handle roughly $10^{4}-10^{5}$ polyatomic molecules. This will be an enormous advance over what has already been done for other systems and is a necessary step toward understanding the fracture/failure of these crystals.

These studies are essential to support efforts to represent the macroscopic effects of crystal fracture and failure. Examples at Los Alamos include work by Dienes, et al, using statistical crack mechanics and work by Brackbill, et al., using modified particle-in-cell FLuid-Implicit Particle (FLIP) methods. Data are needed away from ambient conditions of pressure and temperature. For example, the effect of the viscosity or thermal conductivity of a liquefied explosive at the hot tip of a rapidly propagating crack must be understood. The significant expense and inherent difficulty (or impossibility) of performing experiments capable of providing the required data, and the interest in developing science-based, rather than empirical models, indicate an urgent need for computational approaches.

\section{F. Grain-grain and grain-binder interactions}

This research element centers on first-principles modeling on a larger scale using approaches such as the Discrete Element Method. The question is whether explosive and energetic material behavior is largely determined at the level of grain-grain and grain-binder interactions. The role of the smaller scale simulations and relevant experiments would be to provide the necessary input material properties for the grains and binder materials. These would include the fracture properties of the grains and rheological/constitutive properties of the binders.

Simulations on this scale can help determine if the dynamic response and initiation of energetic materials can be predicted by accurately following stress bridging in the composite granular material, resulting statistically in stress and energy localization and incipient reaction. If this approach succeeds, the problem of predicting the behavior of energetic materials from first principles can be logically separated into uncoupled microscale calculations for grain properties and larger scale calculations of the grain interactions.

G. Aging effects in energetic materials 
Results in the other research elements may be dramatically affected by the aging process. By their very nature, energetic materials are metastable molecules. Therefore, over time, they will decompose, produce gas, and at higher temperatures change molecular composition and structure. These changes lead to changes in the macroscopic behavior of these materials, and can have profound nonlinear effects on systems containing them.

Degradation mechanisms and chemical reactions must be addressed to understand the performance consequences of aging in energetic materials. The effects of aging on transport coefficients are important for other issues as well, such as the outgassing of binders and plasticizers in aging energetic materials. The understanding of fracture/failure of energetic material crystals must include the relationship (largely unknown) between chemical and molecular structure and morphology for aging energetic materials. In addition, the relationship of the composition of aged energetic materials to their performance once detonated must be addressed. 